\title{
Responsiveness of The Countermovement Jump and Handgrip Strength to an Incremental Running Test in Endurance Athletes: Influence of Sex
}

\author{
by \\ Felipe García-Pinillos ${ }^{1}$, Pedro Delgado-Floody², Cristian Martínez-Salazar², \\ Pedro Á. Latorre-Román ${ }^{1}$
}

The present study analyzed the acute effects of an incremental running test on countermovement jump (CMJ) and handgrip strength performance in endurance athletes, considering the effect of post-exercise recovery time and sex. Thirty-three recreationally trained long-distance runners, 20 men and 13 women, participated voluntarily in this study. The participants performed the Léger test, moreover, the CMJ and handgrip strength tests were carried out before and after the running test and during different stages of recovery (at the 1st min of recovery (posttest1), 5th min of recovery (posttest2), and 10th min of recovery (posttest3)). Two-way analysis of variance revealed a significant improvement in the CMJ (pre-posttest1, $p=0.001$ ) and handgrip strength (pre-posttest2, $p=0.017$ ) during recovery time. The Pearson's Chi-2 test showed no significant relationship $(p \geq 0.05)$ between sex and post-activation potentiation (PAP). A linear regression analysis pointed to heart rate recovery as a predictive factor of CMJ improvement (PAP). In conclusion, despite significant fatigue reached during the Léger test, the long-distance runners did not experience an impaired CMJ and handgrip strength performance, either men or women, achieving an improvement $(P A P)$ in posttest conditions. The results obtained showed no significant relationship between sex and PAP. Moreover, significant effect of recovery after running at high intensity on CMJ performance and handgrip strength was found. Finally, the data suggest that PAP condition can be predicted by heart rate recovery in endurance runners.

Key words: long-distance runner, post-activation potentiation, fatigue, recovery time, rapid force.

\section{Introduction}

Previous muscle contractions of skeletal muscle play a pivotal role in the subsequent performance from that muscle group (Kilduff et al., 2007; Wilson et al., 2013). Muscle contractions may acutely decrease or increase force production in a subsequent motor activity: force depression after a previous muscle contraction is known as fatigue, while force enhancement is defined as post-activation potentiation (PAP) (Batista et al., 2007). Any previous muscle activity can trigger both PAP and fatigue mechanisms. Thus, the performance enhancement depends on the prevalence of PAP mechanisms over fatigue mechanisms, which is modulated by several factors including, but not limited to, sociodemographic characteristics of subjects like sex (Russ et al., 2008) or training experience (Kilduff et al., 2007), the recovery time (Gołaś et al., 2016; Kilduff et al., 2008), and the type, intensity as well as volume of the conditioning activity (CA) performed (Gołaś et al., 2016; Wilson et al., 2013).

1 - Faculty of Education Sciences, Department of Didactics of Corporal Expression. University of Jaen. Paraje de Las Lagunillas s/n. D2 Building, Dep. 142. 23071 Jaen (Spain).

2 - Universidad de La Frontera, Faculty of Education, Social Sciences and Humanities. Department of Physical Education, Sport and Recreation. Temuco, Chile. 
Previous studies indicate that not only explosive, shorter and intensive stimuli can be used as a CA, but also sub-maximum and longer or prolonged exercises can cause PAP for subsequent activities (Boullosa and Tuimil, 2009; Boullosa et al., 2011; García-Pinillos et al., 2015; Vuorimaa et al., 2006). Hamada et al. (2000) indicated that endurance training caused, on one hand, a greater amount of phosphorylation of regulatory myosin light chains in slow fibers, and on the other hand, greater resistance to fatigue, which would allow the prevalence of potentiation as well as explain the PAP presence in endurance athletes.

Muscle fatigue is a complex phenomenon resulting from impairment in the neuromuscular system (Allman and Rice, 2002; Russ et al., 2008; Zajac et al., 2015). This impairment is shown as a decline in peak force generating capacity. Greater resistance to fatigue has been associated with sex, less fatigue in women than men (Clark et al., 2005), although these results are not universal (Russ et al., 2008). The results of several studies have suggested that differences in muscle mass, strength (Lanza et al., 2004), and peripheral nervous system excitability (Allman and Rice, 2002; Russ et al., 2008) do not consistently explain sex-related differences in the fatigue of voluntary contractions, particularly during high-intensity intermittent contraction protocols. The role of central activation in sex-related differences in fatigue is equivocal. Previous studies have reported that fatigue resistance of young women compared with young men is accompanied by reduced central activation failure in women (Russ and Kent-Braun, 2003), although other research has shown (Ros et al., 2013) no differences in central activation. In this regard, the presence/absence of supraspinal fatigue (or central fatigue) during running exercises can be determined by changes in the force of muscles not involved in a prolonged running exercise (i.e., handgrip strength test) (Kilduff et al., 2007; Martin et al., 2010; Paavolainen et al., 1999). Likewise, the recovery time between the preload stimuli and subsequent explosive activity is also a controversial topic, with studies reporting optimal recovery periods ranging from 0 to 18.5 minutes (Kilduff et al., 2007, 2008; Wilson et al., 2013).

After a CA protocol (i.e., running exercises), the mechanism of muscular fatigue and
PAP coexist, and thus, the subsequent power output and performance depend on the balance between these two factors. As highlighted before, some previous works have already focused on examining the effect of fatigue on performance in explosive tasks. However, the literature available about the influence of variables such as sex or recovery time is scarce, and as a result, there is no consensus on this matter. It is possible that sex and the recovery period play a key role in that relationship and, more research is needed in this field. Therefore, the aim of this study was to analyze the acute effects of an incremental running test on countermovement jump (CMJ) and handgrip strength performance in endurance athletes, considering the influence of variables such as sex and post-exercise recovery time.

\section{Methods}

\section{Participants}

Thirty-three recreationally trained longdistance runners, 20 men (age $=32 \pm 10$ years, body mass index $[\mathrm{BMI}]=21.6 \pm 2.4 \mathrm{~kg} \cdot \mathrm{m}^{-2}$ and maximal oxygen uptake $\left(\mathrm{VO}_{2 \max }\right)=57 \pm 3 \mathrm{ml} \cdot \mathrm{kg}$ ${ }^{1} \cdot \mathrm{min}^{-1}$ ) and 13 women (age $=28 \pm 7$ years, BMI $=$ $20 \pm 1.5 \mathrm{~kg} \cdot \mathrm{m}^{-2}$ and $\left.\mathrm{VO}_{2 \max }=50 \pm 4 \mathrm{ml} \cdot \mathrm{kg}^{-1} \cdot \mathrm{min}^{-1}\right)$, voluntarily participated in this study. The subjects came from different athletic clubs in Andalusia (Spain), and the assessment protocol was performed during the competitive period. Inclusion criteria were: (a) the subjects were experienced athletes, with a minimum of two year experience of training and competition; (b) the subjects trained regularly and they had no history of injury in the previous three months that would limit training. No alcohol intake was allowed the day before testing, strenuous physical exercise was not allowed for 72 hours prior testing, and food intake was not permitted two hours before the test. After receiving detailed information on the objectives and procedures of the study, each subject signed an informed consent form for participation, which complied with the ethical standards of the World Medical Association Declaration of Helsinki (2013). The study was approved by the Ethics Committee of the University of Jaen (Spain).

Procedures

This study examined the effect of sex and recovery duration on $\mathrm{CMJ}$ and handgrip strength performance after an incremental running test. 
This allowed a comparison to be made between performance at rest (unfatigued condition) and at different stages of recovery (fatigued condition). For this study, an original assessment protocol was used. Nevertheless, the tests performed were included in previous works (Boullosa and Tuimil, 2009; Boullosa et al., 2011; García-Pinillos et al., 2015). The subjects were familiarized with the testing protocol. Participants were tested on an indoor court, individually, on one specific day, and the tests were performed in the same order. Testing was integrated into weekly training schedules, although the participants were advised to avoid strenuous exercise $72 \mathrm{~h}$ before its beginning. Participation involved the execution of the Léger test (Léger et al., 1988). In addition, the CMJ performance and handgrip strength were evaluated before and after the Léger test, as well as at different stages of recovery: at the $1^{\text {st }} \mathrm{min}$ of recovery (posttest1), $5^{\text {th }} \mathrm{min}$ of recovery (posttest2), and $10^{\text {th }}$ min of recovery (posttest3). The participants were encouraged to perform the test with maximum intensity.

At the beginning of the testing day, a body composition analysis through InBody R20 (Biospace Co., Ltd., Seoul, Korea) was carried out. This device measured body mass $(\mathrm{kg})$ and fat content (\%). Body height (m) was determined following a standard procedure with a stadiometer (Seca 222, Hamburg, Germany), and the BMI (weight in $\mathrm{kg} \cdot \mathrm{m}^{-2}$ ) was calculated accordingly. Next, the subjects performed a standardized warm-up, which consisted of five minutes of low-intensity running and five minutes of general exercises. Five minutes after the warm-up, the participants performed the pretest (CMJ and handgrip strength test, in that order). Afterwards, they completed the Léger test and, finally, in the fatigued condition (posttest), they performed the same tests (CMJ and handgrip strength test) during the recovery: at the $1^{\text {st }} \mathrm{min}$ of recovery (posttest1), $5^{\text {th }} \mathrm{min}$ of recovery (posttest2), and $10^{\text {th }}$ min of recovery (posttest3).

Materials and instruments

The Léger test (Léger et al., 1988) consists of running back and forth between two lines $20 \mathrm{~m}$ apart, with running speed determined by audio signals from a pre-recorded $\mathrm{CD}$. The running speed increases at the end of each one-minute stage. The running speed is $8.0 \mathrm{~km} \cdot \mathrm{h}^{-1}$ for the first stage, $9.0 \mathrm{~km} \cdot \mathrm{h}^{-1}$ for the second stage, and thereafter increases by $0.5 \mathrm{~km} \cdot \mathrm{h}^{-1}$ each minute. The test ends with the subjects twice failing to reach the lines at the time indicated by the audio signals, demonstrating inability to keep the required pace. It has been used extensively in various studies with endurance runners (GarcíaPinillos et al., 2016a, 2016b) showing a good correlation with other performance variables (Billat and Koralsztein, 1996). $\mathrm{VO}_{2 \max }$ can be estimated (Léger and Lambert, 1982) through the speed that the participants reached in the last sprint through the following equation: $\mathrm{VO}_{2}(\mathrm{ml} / \mathrm{kg}-$ $\left.{ }^{1} \cdot \mathrm{min}^{-1}\right)=5.857 \mathrm{x}$ velocity $\left(\mathrm{km} \cdot \mathrm{h}^{-1}\right)-19.458$. The heart rate (HR) was controlled during the test through a Garmin Forerunner monitor 405 (Kansas, USA), obtaining the peak HR (HR peak), and the average value (HRmean). In addition, the rating of perceived exertion scale (RPE) (Borg, 1982) was completed by participants before and after the test to register the subjective intensity of exertion.

For assessing vertical jumping ability, the $\mathrm{CMJ}$ test was performed. In each measurement (pretest, posttest 1, 2, and 3) the participants performed 3 trials, separated by $15 \mathrm{~s}$ of recovery. From a standing position, the participants were to dip and immediately jump for maximum height. The takeoff was to be performed as a continuous movement with no observable pause between downward and upward phases. The angular displacement of the knee was normalized so that subjects were required to flex the knees to an angle of approximately 90 degrees, no arm swing was allowed. The obtained values were averaged for the analysis. Jump test performance was recorded using the FreePower Jump Sensorize (Biocorp, Italy) device, which provides the following variables: maximum height of the jump $(\mathrm{m})$, peak force (Pforce; $\mathrm{N} \cdot \mathrm{kg}^{-1}$ ), peak power (Ppower; $\left.\mathrm{W} \cdot \mathrm{kg}^{-1}\right)$, eccentric work $\left(\mathrm{EccW} ; \mathrm{J}^{-k^{-1}}{ }^{-1}\right.$ and concentric work (ConcW; J.kg-1). This device had been previously validated (Picerno et al., 2011). The difference between the CMJ performance at different measurements was calculated and used in subsequent analysis $(\Delta)$.

The handgrip strength test was executed during each measurement (pretest and posttest1, 2 and 3), after vertical jump tests. Two attempts of the handgrip were performed with each hand, lasting $3 \mathrm{~s}$ each, and separated by $60 \mathrm{~s}$ of recovery. The average handgrip strength value of both 
hands was then calculated. To record handgrip strength $(\mathrm{kg})$, a digital hand dynamometer (TKK 5101 Grip D; Takey, Tokyo Japan) was used, adjusting the optimum grip through the calibration formula by Ruiz et al. (2006).

Statistical Analysis

The data were analyzed using SPSS. v.21.0 for Windows (SPSS Inc, Chicago, USA) and the significance level was set at $p<0.05$. The data are shown as descriptive statistics of mean, standard deviation (SD), and percentages (\%). The Kolmogorov-Smirnov test was employed to verify normal distribution of data. The comparison of data (physiological characteristics, body composition and physical fitness of participants) according to sex was performed using the Student's t-test. The comparison of data between measures of the assessment protocol was performed using a two-way mixed $(2 \times 4$ [sex $x$ measurements]) repeated-measures analysis (ANOVA). Pearson correlation analysis was performed including different variables and measures. A linear regression analysis was carried out considering HRrec and $\mathrm{CMJ}$ change $(\triangle \mathrm{CMJ})$ from rest condition to post-exercise condition, at the end of the protocol and the $1^{\text {st }} \mathrm{min}$ of recovery. Finally, a cluster analysis (k-means) was performed by grouping according to whether PAP occurred (RG, responder group) or not (NRG, non-responder group) in relation to $\triangle \mathrm{CMJ}$. A Pearson's Chi-2 test was performed to analyze the relationship between sex and PAP condition.

\section{Results}

Additional information on body composition and physical fitness of the participants is given in Table 1. The Student's ttest showed significant differences $(p<0.05)$ in body composition variables (BMI, muscle mass, fat mass) and physiological variables $\left(\mathrm{VO}_{2 \max }\right.$, $H R_{\text {peak }}$ and $\left.H R_{\text {mean }}\right)$ in relation to sex. No significant differences $(p \geq 0.05)$ were found in the $\mathrm{HR}_{\text {rec }}$ and training background (training experience, weekly mileage and sessions per week) according to sex.

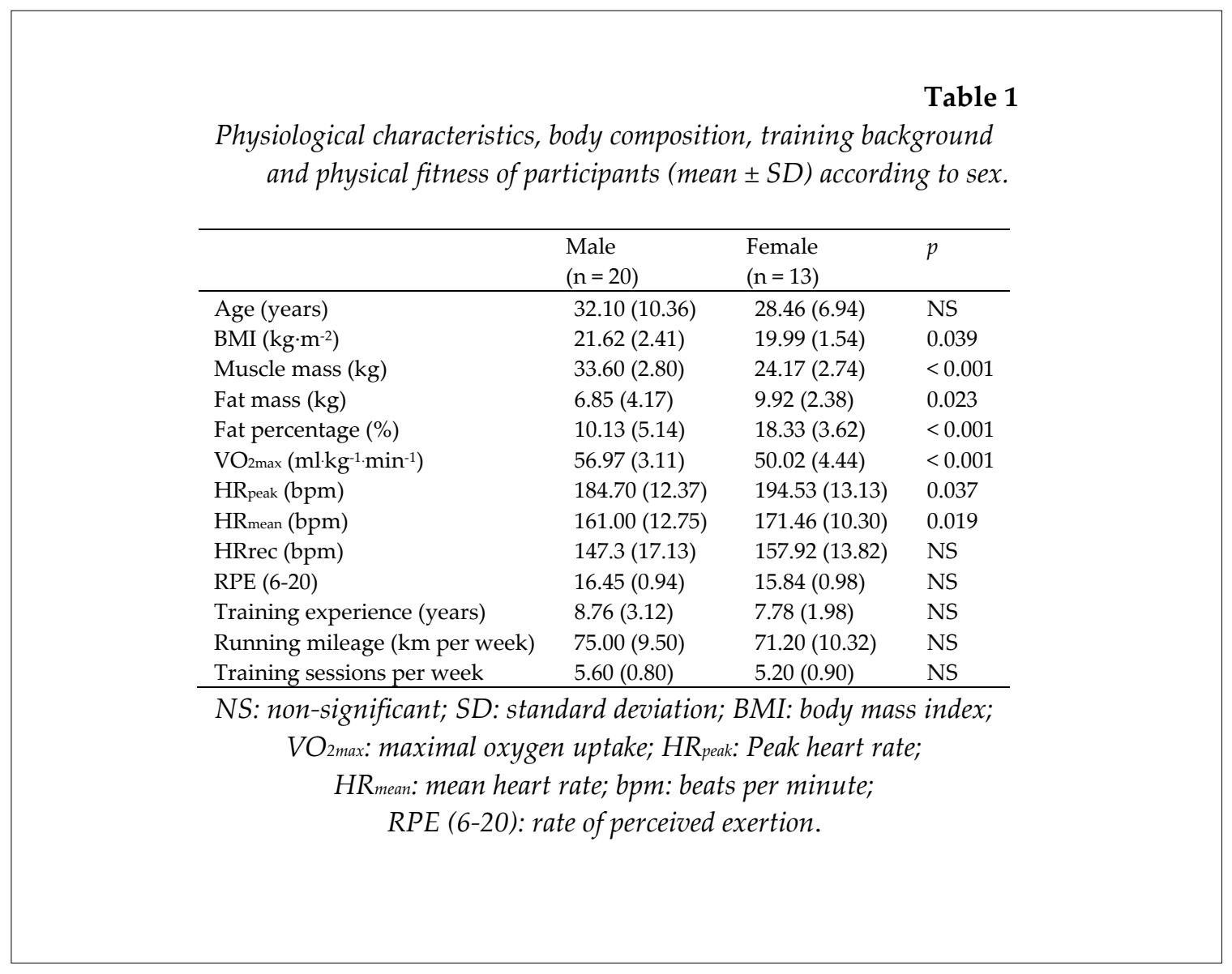


Table 2

Results obtained (mean, SD) in handgrip strength, CMJ and mechanical variables (Pforce, Ppower, EccW and ConcW) at pretest (unfatigued condition) and during the post-exercise recovery (posttest 1,2 and 3), according to sex of participants.

\begin{tabular}{|c|c|c|c|c|c|c|}
\hline & & Pretest & Posttest1 & Posttest2 & Posttest3 & $\begin{array}{l}\mathrm{p}- \\
\text { values\# }\end{array}$ \\
\hline \multirow{4}{*}{$\begin{array}{l}\text { Handgrip Strength } \\
(\mathrm{kg})\end{array}$} & Male & 41.7 (6.2) & 41.8 (6.5) & $42.6(6.7)$ & 40.83 (11.4) & NS \\
\hline & Female & $26.4(4.0)$ & $27.1(3.2)$ & $27.8(3.1)$ & $27.48(2.9)$ & NS \\
\hline & $\begin{array}{l}\mathrm{p}- \\
\text { values§ }\end{array}$ & $<0.001$ & $<0.001$ & $<0.001$ & $<0.001$ & \\
\hline & Total & 35.7 (9.3)a & $36.0(9.1)$ & $\begin{array}{l}36.8 \\
(9.2) \mathrm{a} \\
\end{array}$ & 35.4 (11.1) & 0.017 \\
\hline \multirow[t]{4}{*}{ CMJ (m) } & Male & $\begin{array}{l}0.38 \\
(0.05) \mathrm{a}\end{array}$ & $\begin{array}{l}0.40 \\
(0.05) a, b\end{array}$ & $\begin{array}{l}0.39 \\
(0.05)\end{array}$ & $\begin{array}{l}0.38 \\
(0.05) \mathrm{b}\end{array}$ & 0.007 \\
\hline & Female & $0.33(0.06)$ & $0.34(0.06)$ & $\begin{array}{l}0.33 \\
(0.06)\end{array}$ & $0.32(0.06)$ & NS \\
\hline & $\begin{array}{l}\mathrm{p}- \\
\text { values§ }\end{array}$ & 0.019 & 0.05 & 0.012 & 0.09 & \\
\hline & Total & $\begin{array}{l}0.36 \\
(0.06) \mathrm{a}\end{array}$ & $\begin{array}{l}0.38 \\
(0.06) a, b\end{array}$ & $0.37(0.06)$ & $\begin{array}{l}0.36 \\
(0.06) b\end{array}$ & 0.001 \\
\hline \multirow{4}{*}{ Pforce $\left(\mathrm{N} \cdot \mathrm{kg}^{-1}\right)$} & Male & $16.2(3.2)$ & $15.9(2.2)$ & $16.4(2.5)$ & $15.9(2.0)$ & NS \\
\hline & Female & $12.5(2.0)$ & $12.9(2.1)$ & $12.6(2.2)$ & $12.4(2.2)$ & NS \\
\hline & $\begin{array}{l}\mathrm{p}- \\
\text { values§ }\end{array}$ & 0.01 & $<0.001$ & $<0.001$ & $<0.001$ & \\
\hline & Total & 14.7 (3.3) & 14.7 (2.6) & $14.9(2.9)$ & $14.5(2.7)$ & NS \\
\hline \multirow{4}{*}{ Ppower (W·kg-1) } & Male & $27.3(5.5)$ & 29.5 (5.7) & $28.3(5.3)$ & $28.3(4.6)$ & NS \\
\hline & Female & $19.5(4.7)$ & $22.2(6.3)$ & $20.3(5.9)$ & $19.9(5.3)$ & NS \\
\hline & $\begin{array}{l}\mathrm{p}- \\
\text { values§ }\end{array}$ & $<0.001$ & 0.02 & $<0.001$ & $<0.001$ & \\
\hline & Total & $24.2(6.4) \mathrm{a}^{*}$ & $26.6(6.9) \mathrm{a}^{*}$ & $25.2(6.7)$ & $24.9(6.4)$ & 0.007 \\
\hline \multirow{4}{*}{$\operatorname{EccW}\left(J \cdot \mathrm{kg}^{-1}\right)$} & Male & $-2.2(0.6)$ & $-2.2(0.6)$ & $-2.1(0.6)$ & $-2.2(0.5)$ & NS \\
\hline & Female & $-2.3(0.3)$ & $-2.4(0.4) \mathrm{a}$ & $-2.2(0.4) \mathrm{a}$ & $-2.2(0.4)$ & 0.047 \\
\hline & $\begin{array}{l}\mathrm{p}- \\
\text { values§ }\end{array}$ & NS & NS & NS & NS & \\
\hline & Total & $-2.2(0.5)$ & $-2.2(0.5) \mathrm{a}$ & $-2.1(0.5) a$ & $-2.2(0.5)$ & 0.042 \\
\hline \multirow{4}{*}{ ConcW (J.kg-1) } & Male & $5.9(1.1)$ & $6.2(1.0)$ & $5.9(1.0)$ & $5.9(0.9)$ & NS \\
\hline & Female & $5.5(0.7)$ & $5.7(0.7)$ & $5.5(0.7)$ & $5.4(0.7)$ & NS \\
\hline & $\begin{array}{l}\mathrm{p}- \\
\text { values§ }\end{array}$ & NS & NS & NS & NS & \\
\hline & Total & $5.8(0.9) \mathrm{a}$ & $5.9(0.9) \mathrm{a}, \mathrm{b}$ & $5.8(0.9)$ & $5.7(0.9) b$ & 0.001 \\
\hline
\end{tabular}

\# indicates a within-group (males or females) comparison;

$\S$ indicates a between-group (males vs. females) comparison;

Values with the same letter indicate significant differences within group: $p<0.05$;

${ }^{*} p<0.01$; NS: non-significant; SD: standard deviation; CMJ: countermovement jump;

Pforce: peak force; Ppower: peak power; EccW: eccentric work;

ConcW: concentric work; posttest1: $1^{\text {st }}$ min of recovery;

posttest2: $5^{\text {th }}$ min of recovery; posttes 3 : 10 $0^{\text {th }}$ min of recovery. 


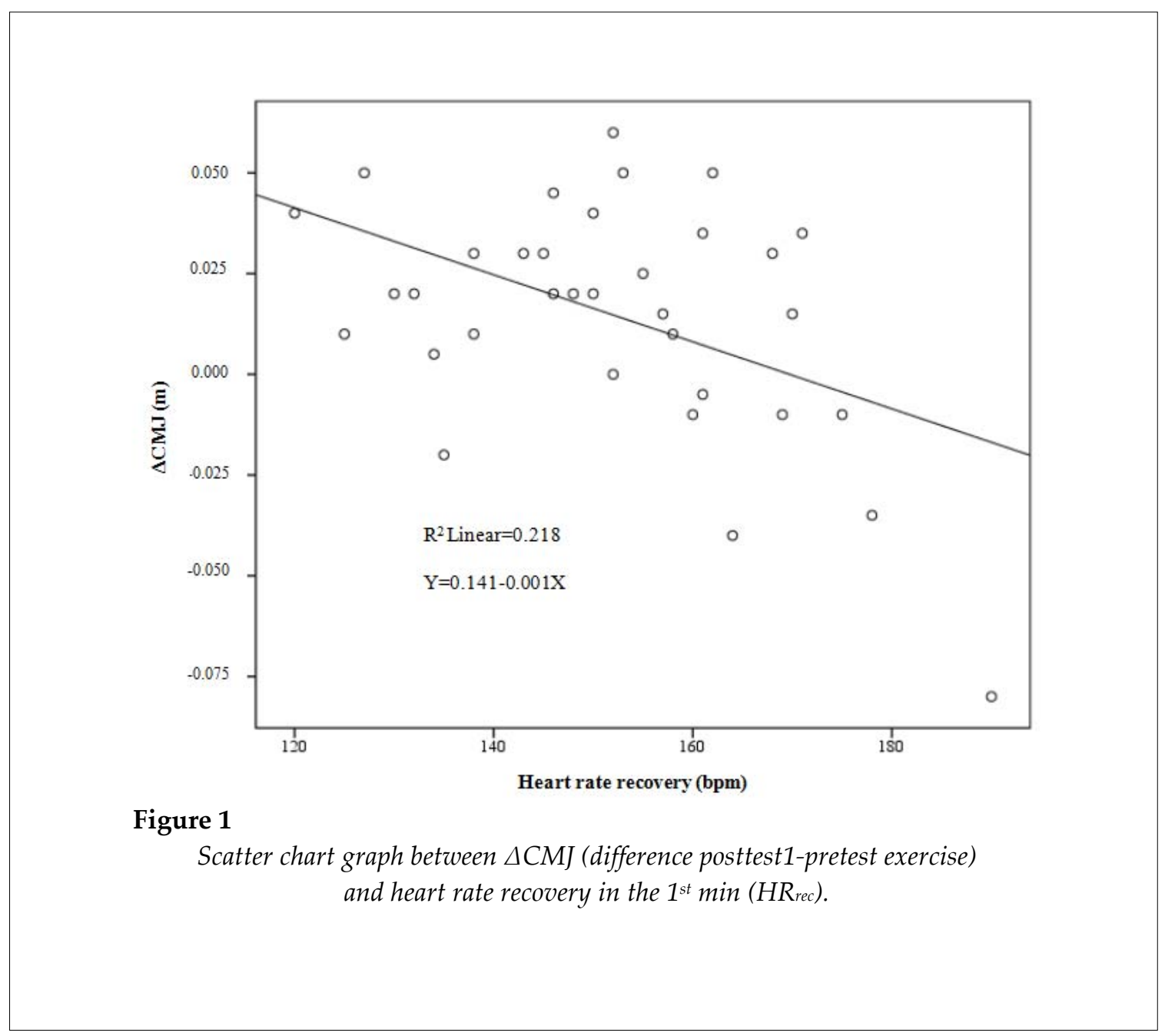

The ANOVA showed significant differences in performance throughout the assessment protocol (Table 2). For the whole group, significant differences in handgrip strength (between pretest-posttest2, $p<0.05$ ), the CMJ (between pretest-posttest1 and posttest1posttest3, $p<0.05)$, and mechanical variables: Ppower $(p=0.007), \operatorname{EccW}(p=0.042)$ and ConcW $(p=0.001)$ were found. According to sex, significant differences in $\mathrm{CMJ}$ performance for men $(p=0.007)$ and EccW for women $(p=0.047)$ were observed.

The Pearson correlation analysis showed significant relationships between $\triangle \mathrm{CMJ}$ (from unfatigued condition, pretest, to posttest 1 ) and HRrec $(R=-0.467, p=0.005)$. HRrec was indicated as a predictive factor of $\triangle \mathrm{CMJ}$, adjusting for age and sex (Figure 1).

Cluster analysis in relation to $\triangle \mathrm{CMJ}$ between pretest and posttest1 was performed. This analysis grouped the participants into 25 who experienced PAP (responder group, RG: 16 men and 9 women) and 8 who did not (non-responder group, NRG: 4 men and 4 women). The Pearson's Chi-2 test showed no significant association $(p \geq$ $0.05)$ between sex and PAP. Considering both groups, a significant difference in handgrip strength ( $p=0.009$ ) was found for the RG between pretest-posttest2, while in the NRG no such a change was observed.

\section{Discussion}

The main aim of this study was to analyze the acute effects of the Léger test performance on the CMJ and handgrip strength test performance in endurance athletes, determining whether fatigue reached would be higher than the PAP effect (impairment of performance) or the opposite (improvement of performance). The major finding of this study was that performance 
in the $\mathrm{CMJ}$ and handgrip strength was not reduced after the Léger test in long-distance runners. In fact, despite significant fatigue, the CMJ performance improved after 1 minute of recovery $(+2 \mathrm{~cm})$ and handgrip strength increased after 5 minutes of recovery $(+1.08 \mathrm{~kg})$, thus PAP occurred. To establish to what extent the subjects reached objective fatigue and to register recovery after the running test, HR values and the RPE scale (Borg, 1982) were used. Immediately after performance of the Léger test, HRpeak for men and women was 184 and 194 bpm, HRmean 161 and $171 \mathrm{bpm}$, and median RPE was 16.45 and 15.84 (very hard), respectively, which could be interpreted as reflecting high levels of fatigue (Borg, 1982; Ros et al., 2013).

The improvement of post-exercise CMJ performance (PAP) registered in this study confirms the results obtained in similar studies conducted previously (Bomfim Lima et al., 2011; Boullosa and Tuimil, 2009; Boullosa et al., 2011; García-Pinillos et al., 2015), where significant differences were found after completion of the running test compared to baseline values. Regarding handgrip strength, as indicated in Paavolainen et al. (1999), the decreased muscular force in those muscles not involved in the exercise (handgrip strength) revealed supraspinal fatigue. Other authors (Kilduff et al., 2007; Martin et al., 2010) examining whether supraspinal fatigue would occur after prolonged exercise, noted the absence of changes in the force of muscles not involved in a prolonged running exercise through measuring handgrip strength. Considering this rationale, the results obtained in this study could indicate that selective supraspinal fatigue does not occur in this type of exercise.

The second aim of this study was to determine the influence of post-exercise recovery time on considered performance variables (CMJ and handgrip strength). This could be assessed clinically whether these tests are sensitive enough to detect when an endurance athlete is in his/her optimal moment of recovery. To date, a limited number of studies examining post-conditioning activity rest periods has yielded varying and often conflicting results. These studies, collectively, suggest that brief $(5 \mathrm{~min})$, moderate $(8-12 \mathrm{~min}$ ) and extensive $(18.5 \mathrm{~min})$ recovery duration may elicit PAP (Gołaś et al., 2016; Kilduff et al., 2007; Wilson et al., 2013). The results obtained in this study show the effect of PAP in vertical jumping ability in the 1 st min posttest $(+2 \mathrm{~cm})$, as well as the capacity for maintaining the initial jumping performance during 10 min posttest. McMahon and Jenkins (2002) further explain this phenomenon. This effect could be caused by a complete recovery of phosphocreatine and would be achieved between the first and second minute of recovery in endurance-trained runners. In this regard, the results obtained in the present study are consistent with the above rationale. The inverse correlation found between $\triangle \mathrm{CMJ}$ (pretestposttest1) and HRrec suggests that the PAP condition is related not only to recovery of phosphocreatine (McMahon and Jenkins, 2002), but also cardiac recovery. Since HRrec has been described as an indicator for functional adaptation in trained subjects in endurance sports (Otsuki et al., 2007), the obtained results might indicate that runner's ability of experiencing PAP after an incremental running exercise is related to training adaptations in endurance athletes. Nevertheless, this finding should be cautiously interpreted and more research is clearly needed to confirm this relationship. These findings differed based on training status, possibly explaining previous discrepancies in the literature. As for the muscles not involved in the CA, the results obtained in this study show a significant improvement in handgrip strength performance 5 min post-exercise $(+1.08 \mathrm{~kg})$ with respect to baseline values. To our knowledge, there are no previous studies on the recovery profile considering muscles not involved during running. The results obtained in this study suggest the association between this fact and the absence of central and supraspinal fatigue after an incremental running test.

The third aim of this study was to analyze the influence of sex on the responsiveness of CMJ and handgrip strength performance to an incremental running test. Our findings indicate that sex is not an influential variable in this protocol. The researchers kept in mind that the study subjects had a similar sports level and constituted a homogenous group. Men presented a higher performance throughout the test, which was consistent with previous studies (Gonzales and Scheuermann, 2007; Ros et al., 2013; Russ et al., 2008). However, the data for male and female subjects show similar trends during the whole 
procedure. The handgrip strength test and CMJ results did not deteriorate despite the high levels of fatigue reported after the Léger test. Significant differences could not be seen in handgrip strength in men nor in women. For the CMJ, it should be noted that men performed significantly better in this test between pretest and posttest1 $(+2 \mathrm{~cm})$, while results for women showed no significant improvement $(+1 \mathrm{~cm})$. In this context, the results obtained by previous studies are controversial. A previous study found sex difference in soccer players after intermittent sprinting bouts, and the authors explained this by showing the differences in muscle mass, muscle morphology, muscle fiber type, and substrate use (Laurent et al., 2010). In contrast, results consistent with the present study were reported by previous works (Gonzales and Scheuermann, 2007; Russ et al., 2008; Wilson et al., 2013), in which no differences between sex in different conditions were observed. Such discrepancies in the findings of the aforementioned studies could be attributable to the different muscles being studied, different tests or protocols used or, simply, to different characteristics of the study subjects.

Based on previous studies (Boullosa and Tuimil, 2009; Boullosa et al., 2011; García-Pinillos et al., 2015) and for a better understanding of the results obtained, we decided to incorporate cluster analysis, as members of the same cluster are likely to have more similar responses. Two clusters of endurance athletes were obtained from the different magnitude of the $\Delta \mathrm{CMJ}$. As mentioned above, these clusters were categorized as responders ( $R G, \mathrm{n}=25 ; 16$ men and 9 women) and non-responders (NRG, $\mathrm{n}=8 ; 4$ men and 4 women). Based on this analysis, we can strengthen our previous conclusions: men and women are divided equally between the RG and NRG and therefore, experiencing PAP is not associated with sex; additionally, the RG showed an increase in handgrip strength under fatigue (posttest2), while in the NRG no such a change was observed.

In conclusion, our findings show that PAP occurs after an incremental running exercise (Léger test) in endurance athletes. Despite significant fatigue reached during the running protocol, experienced female and male longdistance runners did not decrease their CMJ and handgrip strength performance achieving an improvement (PAP) in the posttest condition. A significant effect of recovery after running at high intensity on CMJ performance and handgrip strength was found. Specifically, the CMJ performance improved at the 1st min posttest, while handgrip strength performance increased after $5 \mathrm{~min}$ of recovery. Moreover, the data suggest that HRrec can identify the capacity of PAP in long-distance runners. Lastly, the obtained results show no significant association between sex and PAP.

\section{Acknowledgements}

This study received no financial support. However, the authors would like to thank to "Club Atletismo Unicaja (Jaén)" and "Club Atletismo Media Legua (Baena)" for their support and collaboration, and also to all those athletes who contributed disinterestedly in this research.

\section{References}

Allman BL, Rice CL. Neuromuscular fatigue and aging: central and peripheral factors. Muscle $\mathcal{E}$ Nerve, 2002; 25(6):785-96

Batista MA, Ugrinowitsch C, Roschel H, Lotufo R, Ricard MD, Tricoli VA. Intermittent exercise as a conditioning activity to induce postactivation potentiation. J Strength Cond Res, 2007; 21(3):837-40

Billat LV, Koralsztein JP. Significance of the Velocity at VO2max and Time to Exhaustion at this Velocity. Sport Med, 1996; 22(2): 90-108

Bomfim Lima JC, Popp Marin D, Barquilha G, Ortega da Silva L, Puggina EF, Pithon-Curi TC, Hirabara SM. Acute effects of drop jump potentiation protocol on sprint and countermovement vertical jump. Human Mov, 2011; 12(4):324-330

Borg G. Psychophysical bases of perceived exertion. Med Sci Sports Exerc, 1982; 14(5): 377-381

Boullosa DA, Tuimil JL. Postactivation potentiation in distance runners after two different field running protocols. J Strength Cond Res, 2009; 23(5):1560-1565

Boullosa DA, Tuimil JL, Alegre LM, Iglesias E, Lusquiños F. Concurrent fatigue and potentiation in 
endurance athletes. Int J Sports Physiol Perf, 2011; 6(1):82-93

Clark BC, Collier SR, Manini TM, Ploutz-Snyder LL. Sex differences in muscle fatigability and activation patterns of the human quadriceps femoris. Eur J Appl Physiol, 2005; 94(1-2):196-206

García-Pinillos F, Molina-Molina A, Latorre-Román PÁ. Impact of an incremental running test on jumping kinematics in endurance runners: can jumping kinematic explain the post-activation potentiation phenomenon? Sports Biomechanics / International Society of Biomechanics in Sports, 2016; 15(2), 103-115.

García-Pinillos F, Serrano-Huete V, Salas-Sánchez J, Ortega-García R, Gómez-Rodríguez FJ, Latorre-Román PA. Influence of sex, athletic performance and age differences on the acute cardiovascular and thermoregulatory response to incremental test in endurance runners. Science $\mathcal{E}$ Sports, 2016; 31(5): 123129

García-Pinillos F, Soto-Hermoso VM, Latorre-Román PA. Acute effects of extended interval training on countermovement jump and handgrip strength performance in endurance athletes: postactivation potentiation. J Strength Cond Res, 2015; 29(1):11-21

Gołaś A, Maszczyk A, Zajac A, Mikołajec K, Stastny P. Optimizing post activation potentiation for explosive activities in competitive sports. J Hum Kinet, 2016; 52(1): 95-106

Gonzales JU, Scheuermann BW. Absence of gender differences in the fatigability of the forearm muscles during intermittent isometric handgrip exercise. J Sports Sci Med, 2007; 6(1): 98-105

Hamada T, Sale DG, Macdougall JD. Postactivation potentiation in endurance-trained male athletes. Med Sci Sports Ex, 2000; 32: 403-411

Kilduff LP, Bevan HR, Kingsley MIC, Owen NJ, Bennett MA, Bunce PJ, Hore AM, Maw JR, Cunningham DJ. Postactivation potentiation in professional rugby players: optimal recovery. J Strength Cond Res, 2007; 21(4): 1134-8

Kilduff LP, Owen N, Bevan H, Bennett M, Kingsley MIC, Cunningham D. Influence of recovery time on post-activation potentiation in professional rugby players. J Sports Sci, 2008; 26(8):795-802

Lanza IR, Russ DW, Kent-Braun JA. Age-related enhancement of fatigue resistance is evident in men during both isometric and dynamic tasks. J Appl Physiol, 2004; 97(3): 967-75

Laurent CM, Green JM, Bishop PA, Sjökvist J, Schumacker RE, Richardson MT, Curtner-Smith M. Effect of gender on fatigue and recovery following maximal intensity repeated sprint performance. J Sports Med Phys Fitness, 2010; 50(3): 243-53

Léger LA, Lambert J, Leger LA, Lambert J. A maximal multistage 20-m shuttle run test to predict VO2 max. Eur J Appl Physiol Occupat Physiol, 1982; 49(1): 1-12

Léger LA, Mercier D, Gadoury C, Lambert J. The multistage 20 metre shuttle run test for aerobic fitness. J Sports Sci, 1988; 6(2): 93-101

Martin V, Kerhervé H, Messonnier LA, Banfi JC, Geyssant A, Bonnefoy R, Feasson L, Millet GY. Central and peripheral contributions to neuromuscular fatigue induced by a 24-h treadmill run. J Appl Physiol, 2010; 108(5): 1224-33

McMahon S, Jenkins D. Factors affecting the rate of phosphocreatine resynthesis following intense exercise. Sports Med, 2002; 32(12): 761-84

Otsuki T, Maeda S, Iemitsu M, Saito Y, Tanimura Y, Sugawara J, Ajisaka R, Miyauchi T. Postexercise heart rate recovery accelerates in strength-trained athletes. Med Sci Sports Exerc, 2007; 39(2): 365-70

Paavolainen L, Nummela A, Rusko H, Häkkinen K. Neuromuscular characteristics and fatigue during $10 \mathrm{~km}$ running. Int J Sports Med, 1999; 20(8): 516-21

Picerno P, Camomilla V, Capranica L. Countermovement jump performance assessment using a wearable 3D inertial measurement unit. J Sports Sci, 2011; 29(2): 139-46

Ros A, Holm S, Fridén C, Heijne AL. Responsiveness of the one-leg hop test and the square hop test to fatiguing intermittent aerobic work and subsequent recovery. J Strength Cond Res, 2013; 27(4): 988-994

Ruiz JR, España-Romero V, Ortega FB, Sjöström M, Castillo MJ, Gutierrez A. Hand Span Influences Optimal Grip Span in Male and Female Teenagers. J Hand Surg, 2006; 31(8):1367-1372

Russ DW, Kent-Braun JA. Sex differences in human skeletal muscle fatigue are eliminated under ischemic conditions. J Appl Physiol, 2003; 94(6):2414-2422

Russ DW, Towse TF, Wigmore DM, Lanza IR, Kent-Braun JA. Contrasting influences of age and sex on 
muscle fatigue. Med Sci Sports Exerc, 2008; 40(2): 234-41

Vuorimaa T, Virlander R, Kurkilahti P, Vasankari T, Häkkinen K. Acute changes in muscle activation and leg extension performance after different running exercises in elite long distance runners. Eur J Appl Physiol, 2006; 96(3): 282-91

Wilson J, Duncan N, Marin P, Brown L, Loenneke J, Wilson S, Jo E, Lowery RP, Ugrinowitsch C. Metaanalysis of Postactivation Potentiation and Power: Effects of Conditioning Activity, Volume, Gender, Rest Periods, and Training Status. J Strength Cond Res, 2013; 27(3): 854-859

Zając A, Chalimoniuk M, Maszczyk A, Gołaś A, Lngfort J. Central and Peripheral Fatigue During Resistance Exercise A Critical Review. J Hum Kinet. 2015; 49(1): 159-169

\section{Corresponding author:}

\section{Felipe García-Pinillos.}

Faculty of Education Sciences. Department of Didactics of Corporal Expression. University of Jaén (Spain). Campus de las Lagunillas s/n, 23071, Jaén (Spain).

Tel: (+34) 660062066, (+34) 953212710.

E-mail: fegarpi@gmail.com 